\title{
The Pathophysiology and Progression of Hip Osteoarthritis Accompanied with Joint Pain are Potentially Due to Bone Alterations - Follow-up Study of Hip OA Patients
}

\author{
Mikio Kamimura ${ }^{1}$, Yukio Nakamura ${ }^{*}$, , Shigeharu Uchiyama ${ }^{2}$, Shota Ikegami ${ }^{2}$, Keijiro Mukaiyama $^{2}$ \\ and Hiroyuki Kato ${ }^{2}$ \\ ${ }^{1}$ Center of Osteoporosis and Spinal Disorders: Kamimura Orthopaedic Clinic, Matsumoto 399-0021, Japan \\ ${ }^{2}$ Department of Orthopaedic Surgery, Shinshu University School of Medicine, Asahi 3-1-1, Matsumoto 390-8621, Japan
}

\begin{abstract}
Objectives: This study examined hip osteoarthritis (OA) patients with joint pain and accompanying signal changes detected by magnetic resonance imaging (MRI).

Methods: A total of 19 hip OA patients with suddenly occurring or worsening pain regardless of Kellgren-Lawrence grading were enrolled. The patients were monitored using MRI, plain radiographs, and the Denis pain scale for a minimum of 6 months. The patients were classified into 2 groups: those whose pain improved during conservative treatment (Group A) and those whose pain persisted (Group B).

Results: Joint pain disappeared or was markedly improved in all 10 cases in Group A. Radiographic OA progression occurred in 7 of 8 cases with available radiographs. Hip MRI was performed on 7 of 10 patients, among whom bone signal changes disappeared in 6 patients. One patient exhibited persisting bone signal alterations although joint pain had completely disappeared. In Group B, joint pain remained in all 9 cases. Radiographic OA progression occurred in 8 of 9 cases, and local (4 cases) or broad (5 cases) bone signal alterations were present in end-point MRI examinations. Two patients exhibited different regional MRI bone signal changes (local or broad) at the end of follow-up. The mean age of Group B was significantly higher than that of Group A.

Conclusion: This study uncovered the following observations: 1) hip OA with joint pain had bone alterations that were detectable by MRI, 2) these bone alterations disappeared when joint pain improved, 3) bone alterations remained when joint pain continued, and 4) radiographic OA progressed to a more advanced stage over a short time period. These findings indicate that the pathophysiology of OA, joint pain, and OA progression may primarily be due to bone changes.
\end{abstract}

Keywords: Bone alteration, hip, joint pain, MRI, OA.

\section{INTRODUCTION}

Osteoarthritis (OA) is the most common type of chronic joint affliction [1]. Recently, OA has been considered to be a whole-joint disease process, for which whole-organ, semiquantitative assessment using magnetic resonance imaging (MRI) is currently available [2, 3]. Accordingly, MRI examination is becoming an important tool in the evaluation of OA.

Our group has published several reports on the relationships among joint pain, MRI examination, and radiographic evaluation of OA progression [4, 5]. In our first study, advanced OA patients with joint pain exhibited noticeable bone signal changes in MRI whereas those without joint pain did not. Therefore, we suggested that the pathophysiology of joint pain was primarily due to bone

*Address correspondence to this author at the Department of Orthopaedic Surgery, Shinshu University School of Medicine, Asahi 3-1-1, Matsumoto 390-8621, Japan; Tel.: +81-263-37-2659; Fax: +81-263-35-8844;

E-mail: yxn14@aol.jp alterations [4]. In our second report, early-stage OA patients with joint pain initially displayed bone signal changes in MRI images. Afterwards, OA progressed radiographically while joint pain substantially improved. These findings also implied that the cause of joint pain and OA progression was bone alterations [5].

Guermazi et al. described the existence of OA-associated lesions detected by MRI without obvious plain radiographic features based on a report by Framingham. However, a weakness of their study was that it did not include further imaging evaluation [2]. Based these results, it appears necessary for 1) observation of such patients over a longer time period and 2) analysis of the relationship between signal changes seen in MRI and joint pain. We currently perform MRI to evaluate joints when there is a discrepancy between the degree of joint pain and radiographic findings.

This study reports on the clinical outcomes of hip OA patients presenting with joint pain as evaluated by plain radiographs, MRI, and the Denis pain scale over a minimum 6-month follow-up period. 


\section{PATIENTS AND METHODS}

A total of 19 patients with hip OA presented at our institution with sudden severe or worsened joint pain. Plain radiographs were taken at the first visit and MRI was performed within a month of pain onset. Conservative treatment was given to all patients and plain radiographs were taken 3 months afterwards. Final re-assessment was carried out via MRI examination following a minimum of 6 months after pain onset.

Pain was assessed based on the scale established by Denis [6]: P1, no pain; P2, occasional minimum pain with no need for medication; P3, moderate pain with occasional medication, but no interruption of work or significant changes in activities of daily living (ADL); P4, moderate to severe pain with frequent medication and occasional absence from work or significant change in ADL; P5, constant or severe incapacitating pain requiring chronic medication.

Bone mineral density (BMD) was measured using a Dual-energy X-ray Absorption (DXA) fan-beam bone densitometer (Lunar Prodigy; GE Healthcare, Waukesha, WI, USA) at the L1-4 levels of the posteroanterior spine and bilateral proximal femur. MRI acquisition conditions were as follows for coronal and sagittal views using T1-weighted imaging (T1W): TR: 530 (SI), FA: 90 (SI), and TE: 14 (SI) and for hip skeletal survey using short $\tau$ inversion recovery (STIR): TR: 5010 (SI), FA: 180 (SI), TE: 71 (SI), and TI: 150 (SI). Magnetic field strength was $1.5 \mathrm{~T}$ (SI). Bone signal changes were judged to be present only when the changes were detected by both T1W and STIR.

All patients were prescribed non-steroidal antiinflammatory drugs (NSAIDs) and/or tramadol for joint pain and were instructed to take medication when needed. Patients were generally in good health and did not have any serious underlying conditions. The current study was approved by the institutional ethics committee and informed written consent was obtained from all subjects.

In total, we examined the data of 19 hip joints of 19 patients with primary hip OA that were classified from Kellgren-Laurence (KL) grading I to IV [7]. All patients complained of Denis pain scale P4 at presentation [6]. We then divided the cohort into patients whose pain almost completely disappeared ( $\mathrm{P} 0$ or $\mathrm{P} 1)$, thereby requiring no further analgesic treatment (Group A), and patients whose persistent pain (P2, P3, or P4) necessitated continued management (Group B).

\section{RESULTS}

\section{Clinical Findings}

Group A consisted of 2 men and 8 women with an average age (years), height $(\mathrm{cm})$, body weight $(\mathrm{kg})$, and body mass index (BMI) $\left(\mathrm{kg} / \mathrm{m}^{2}\right)$ of 63.8, 157.1, 55.2, and 22.0, respectively (Table $\mathbf{1}$ ). The average T-scores for L1-4 and the non-affected hip were -1.4 and -1.3 , respectively. Six patients had undergone bisphosphonate (BP) treatment prior to this study. Group B contained 1 man and 8 women with an average age (years), height $(\mathrm{cm})$, body weight $(\mathrm{kg})$, and BMI $\left(\mathrm{kg} / \mathrm{m}^{2}\right)$ of $73.1,152.3,51.9$, and 22.0 , respectively.
The average T-scores for L1-4 and the non-affected hip were -1.3 and -1.2 , respectively. There were 4 patients who had undergone BP treatment and 1 patient who had received selective estrogen receptor modulator treatment prior to this study.

Table 1. Clinical Parameters of Group A and Group B. BMI: body mass index; BMD: bone mineral density.

\begin{tabular}{|c|c|c|}
\hline & Group A & Group B \\
\hline & Mean & Mean \\
\hline \hline Age (years) & 63.8 & 73.1 \\
\hline Gender (F:M) & $8: 2$ & $8: 1$ \\
\hline Height (cm) & 157.1 & 152.3 \\
\hline Weight (kg) & 55.2 & 51.9 \\
\hline BMI (kg/m ${ }^{2}$ ) & 22.0 & 22.0 \\
\hline BMD (L1-4) (T-Score) & -1.4 & -1.3 \\
\hline BMD (Non-affected Hip) (T-Score) & -1.3 & -1.2 \\
\hline
\end{tabular}

There were no statistically significant differences among clinical parameters between the groups. However, the mean age of Group B was higher than that of Group A ( $<<0.05$, Welch's t-test) (Table 1).

\section{Joint Pain and Radiographic and MRI Evaluation}

In Group $\mathrm{A}$, joint pain completely disappeared $(\mathrm{P} 0)$ in 8 of 10 cases, among which 2 cases did not return for further radiographic evaluation and 1 did not undergo final MRI examination (Table 2). Slight joint pain (P1) remained in 2 patients. In Group B, conservative treatment with analgesics was continuously prescribed throughout the study period. MRI and radiographic data were obtained for all patients (Table 3).

In Group A, radiographic OA worsened in 7 of 8 analyzed cases, with 1 case unchanged (Table 2). KL grading changes were I to II in 4 cases, II to II with increased joint space narrowing in 2 cases, and III to IV in 1 case. There were no instances of OA progression of 2 grades or more. Hip MRI was performed in 7 of 10 cases, among which bone signal changes disappeared in 6 . One patient exhibited continuous bone signal alterations without any joint pain. In this patient (case 3), signal changes appeared as sclerotic alterations surrounding a bone cyst in plain radiographs.

In Group $\mathrm{B}$, radiographic $\mathrm{OA}$ progression occurred in 8 of 9 cases (Table 3). As in Group A, 1 case did not progress to more advanced stage OA. KL grading changes were I to I with increased joint space narrowing in 2 cases, I to III in 3 cases, II to II with increased joint space narrowing in 1 case, and II to III in 2 cases. Three of 8 cases $(37.5 \%)$ worsened by $2 \mathrm{KL}$ grades in this group. Local or broad MRI bone signal alterations remained in all 9 cases. Whereas 7 cases showed similar regional signal changes (local to local in 3 cases and broad to broad in 4 cases) at 6 months after pain onset, the remaining 2 cases exhibited different regional bone signal alterations (broad to local and local to broad in 1 case each). 
Table 2. Profiles of 10 Joints of 10 Patients whose Pain Improved during Treatment. JS: Joint Space.

\begin{tabular}{|c|c|c|c|c|c|c|c|c|}
\hline Group A & $\begin{array}{c}\text { Age } \\
\text { (Years) }\end{array}$ & Gender & $\begin{array}{l}\text { Pain at } \\
\text { Latest }\end{array}$ & $\begin{array}{l}\text { MRI at } \\
\text { Onset }\end{array}$ & $\begin{array}{c}\text { MRI } \\
\text { 6M After Onset }\end{array}$ & $\begin{array}{l}\text { KL Grading } \\
\text { at Onset }\end{array}$ & $\begin{array}{l}\text { KL Grading } \\
\text { 3M After Onset }\end{array}$ & $\begin{array}{c}\text { Radiographic } \\
\text { Progression }\end{array}$ \\
\hline 1 & 72 & $\mathrm{~F}$ & P0 & Local & No & I & II & Yes \\
\hline 2 & 72 & $\mathrm{~F}$ & P0 & Local & No & I & II & Yes \\
\hline 3 & 67 & $\mathrm{~F}$ & P0 & Broad & No & II & II: More JS narrowing & Yes \\
\hline 4 & 65 & $\mathrm{~F}$ & P0 & Broad & No & I & II & Yes \\
\hline 5 & 62 & $\mathrm{~F}$ & P0 & Broad & Broad & III & IV & Yes \\
\hline 6 & 56 & M & $\mathrm{P} 0$ & Broad & Not yet (no pain) & II & - & Unknown \\
\hline 7 & 59 & M & P0 & Local & Not yet (no pain) & I & I & No \\
\hline 8 & 55 & $\mathrm{~F}$ & P0 & Broad & Not yet (no pain) & III & - & Unknown \\
\hline 9 & 63 & $\mathrm{~F}$ & $\mathrm{P} 1$ & Broad & No & I & II & Yes \\
\hline 10 & 67 & $\mathrm{~F}$ & $\mathrm{P} 1$ & Local & No & II & II: More JS narrowing & Yes \\
\hline Average & 63.8 & & & & & & & \\
\hline
\end{tabular}

Table 3. Profiles of 9 Joints of 9 Patients whose Pain Persisted during Treatment.

\begin{tabular}{|c|c|c|c|c|c|c|c|c|}
\hline Group B & $\begin{array}{c}\text { Age } \\
\text { (Years) }\end{array}$ & Gender & $\begin{array}{l}\text { Pain at } \\
\text { Latest }\end{array}$ & $\begin{array}{c}\text { MRI at } \\
\text { Onset }\end{array}$ & $\begin{array}{c}\text { MRI } \\
\text { 6M After } \\
\text { Onset }\end{array}$ & $\begin{array}{l}\text { KL Grading } \\
\text { at Onset }\end{array}$ & $\begin{array}{c}\text { KL Grading } \\
\text { 3M After Onset }\end{array}$ & $\begin{array}{c}\text { Radiographic } \\
\text { Progression }\end{array}$ \\
\hline 1 & 71 & $\mathrm{~F}$ & $\mathrm{P} 2$ & Local & Local & I & III & Yes \\
\hline 2 & 76 & $\mathrm{~F}$ & $\mathrm{P} 2$ & Local & Local & II & II & No \\
\hline 3 & 68 & $\mathrm{~F}$ & $\mathrm{P} 2$ & Local & Local & I & I: More JS narrowing & Yes \\
\hline 4 & 75 & $\mathrm{~F}$ & $\mathrm{P} 2$ & Broad & Broad & II & III & Yes \\
\hline 5 & 76 & $\mathrm{~F}$ & P3 & Broad & Local & II & II: More JS narrowing & Yes \\
\hline 6 & 59 & $\mathrm{~F}$ & P3 & Broad & Broad & I & III & Yes \\
\hline 7 & 69 & $\mathrm{~F}$ & P3 & Broad & Broad & II & III & Yes \\
\hline 8 & 88 & M & P4 & Broad & Broad & I & III & Yes \\
\hline 9 & 76 & $\mathrm{~F}$ & P4 & Local & Broad & I & I: More JS narrowing & Yes \\
\hline Average & 73.1 & & & & & & & \\
\hline
\end{tabular}

\section{SELECTED CASES}

\section{Patient 1}

A 76-year-old woman presented complaining of severe right hip joint pain. Plain radiographs of the affected joint showed mild OA of KL grading I (Fig. 1a). Within a month after pain onset, hip MRI displayed broad and heterogeneous low intensity in T1W and high intensity in STIR images (Fig. 1b, c). At more than 6 months after onset, hip MRI disclosed a local signal (Fig. 1d, e) and plain radiographs revealed progressed OA of KL grading II (Fig. 1f). Since her joint pain persisted, she ultimately underwent right hip joint replacement surgery.

\section{Patient 2}

A 63-year-old woman presented with the chief complaint of severe right hip joint pain. Plain radiographs of the affected joint showed moderate OA of KL grading II (Fig. 2a). Within a month after pain onset, hip MRI displayed broad and heterogeneous low intensity in $\mathrm{T} 1 \mathrm{~W}$ and high intensity in STIR images (Fig. 2b, c). At more than 6 months after onset, hip MRI showed no signal changes (Fig. 2d-f) and plain radiographs revealed increased joint space narrowing and KL grading II (Fig. 2f). Her joint pain had disappeared by that time.

\section{Patient 3}

A 72-year-old woman presented complaining of severe right hip joint pain. Plain radiographs of the affected joint showed moderate OA of KL grading III (Fig. 3a). Within a month after pain onset, hip MRI displayed broad and heterogeneous low intensity in T1W and high intensity in STIR images (data not shown). At more than 6 months after onset, hip MRI images remained similar and sclerotic changes around a cyst in the right proximal femoral head were evident (Fig. 3b, c). Plain radiographs revealed progressed OA of KL grading IV (Fig. 3d). Her joint pain had disappeared by that time. 


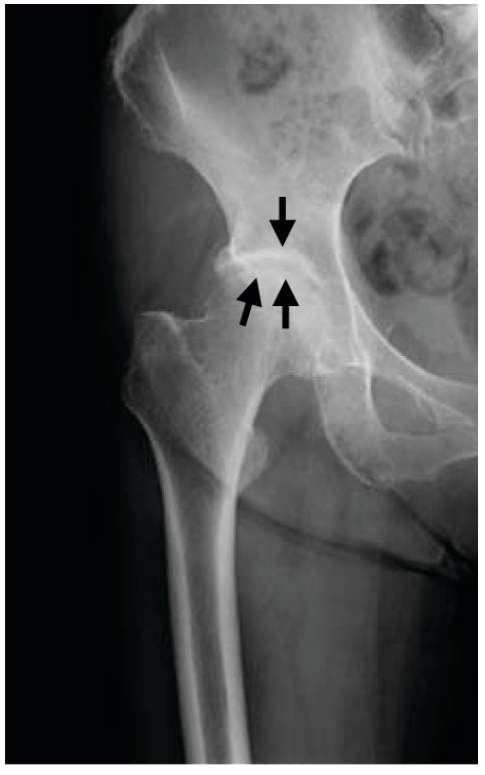

Fig. (1a). Plain radiograph taken at the onset of severe right hip joint pain showing KL grading I hip OA. Joint space narrowing is seen in the right hip joint (black arrows).

(b)

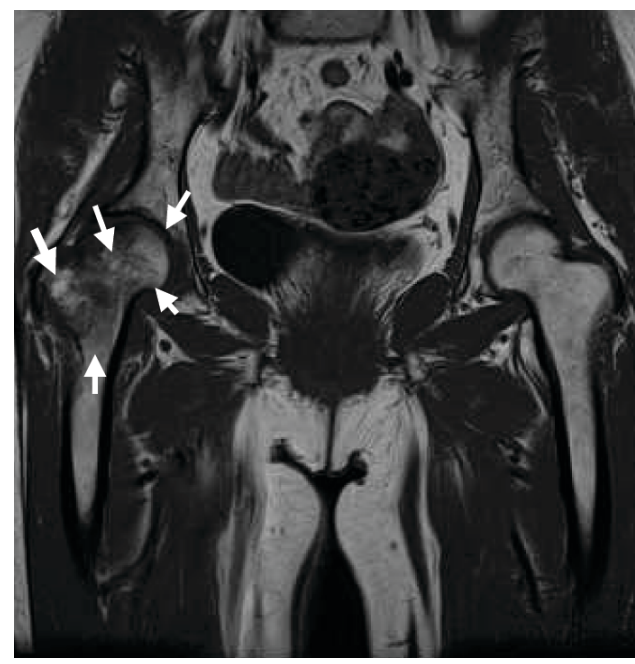

(c)

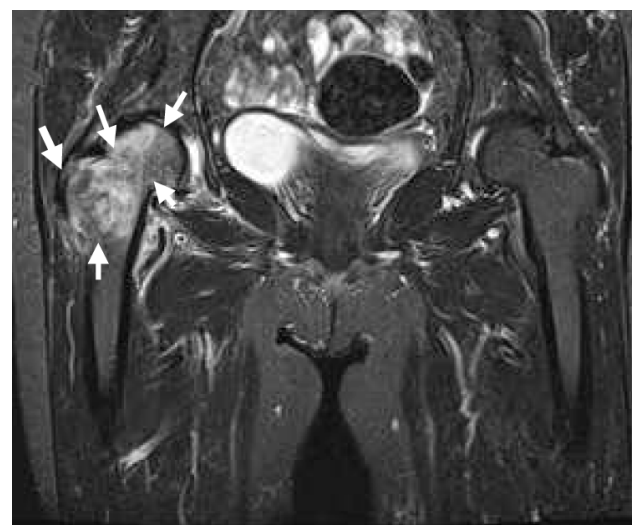

Fig. $(\mathbf{1 b}, \mathbf{c})$. Within a month after pain onset, MRI reveals broad signal changes in the right proximal femur. Broad and heterogeneous low intensity in T1W (b) and high intensity in STIR (c) images are observed (white arrows). (d)

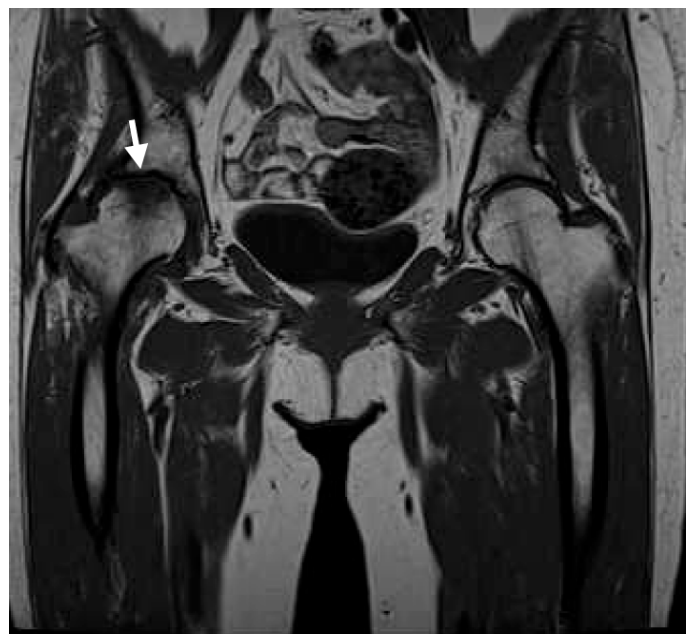

(e)

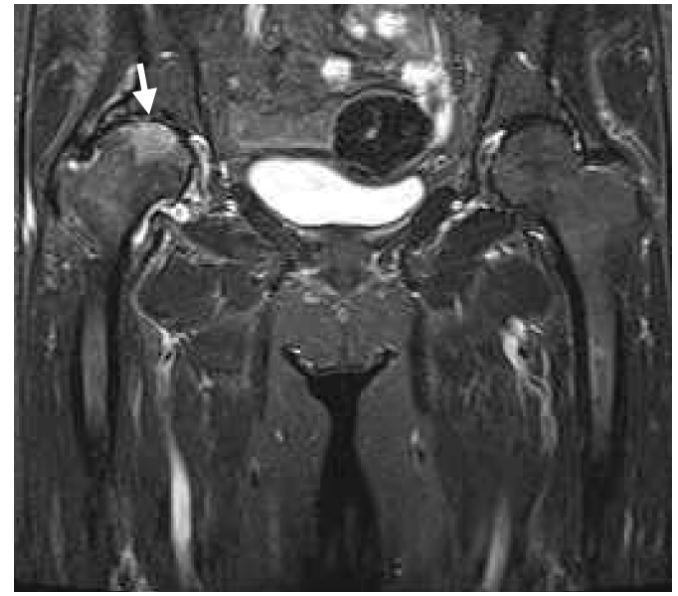

Fig. (1d, e). At more than 6 months after pain onset, MRI reveals local signal changes in the loading parts of the right femoral head in T1W (d) and STIR (e) images (white arrows).

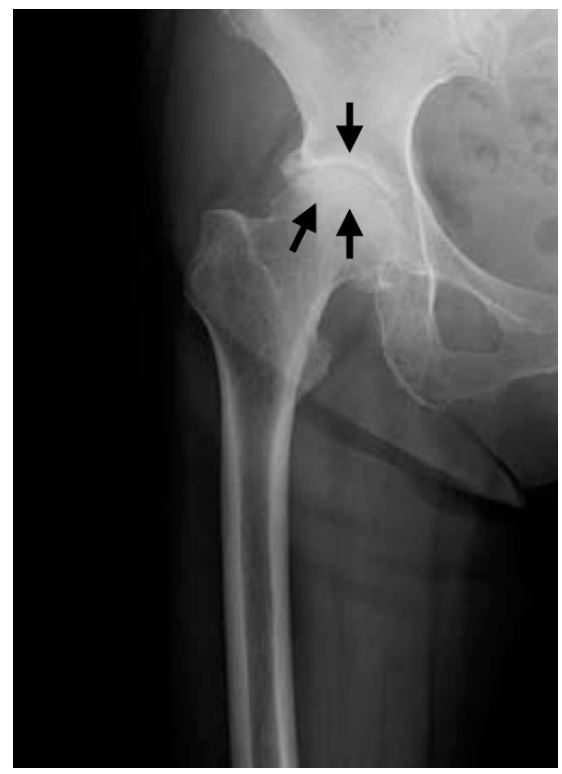

Fig. (1f). Plain radiograph taken after the right hip joint pain had disappeared showing KL grading II hip OA. Increased joint space narrowing is seen in the right hip joint (black arrows). 


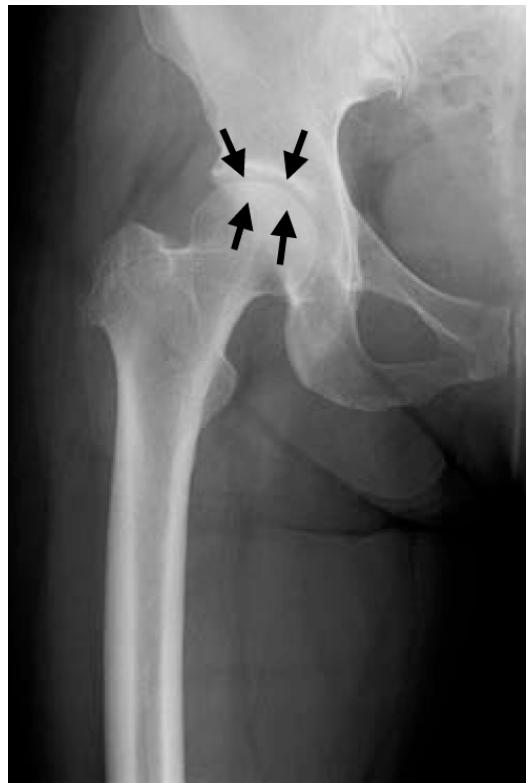

Fig. (2a). Plain radiograph taken at the onset of severe right hip joint pain showing KL grading II hip OA. Joint space narrowing is seen in the right hip joint (black arrows).

(b)

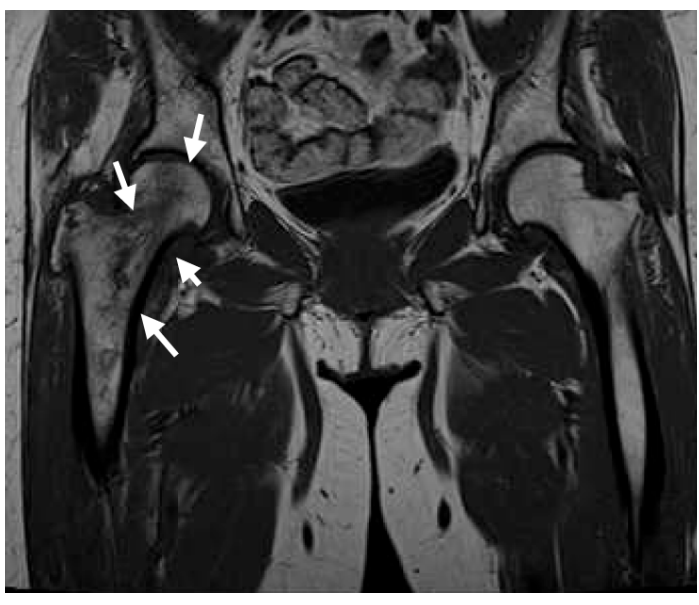

(c)

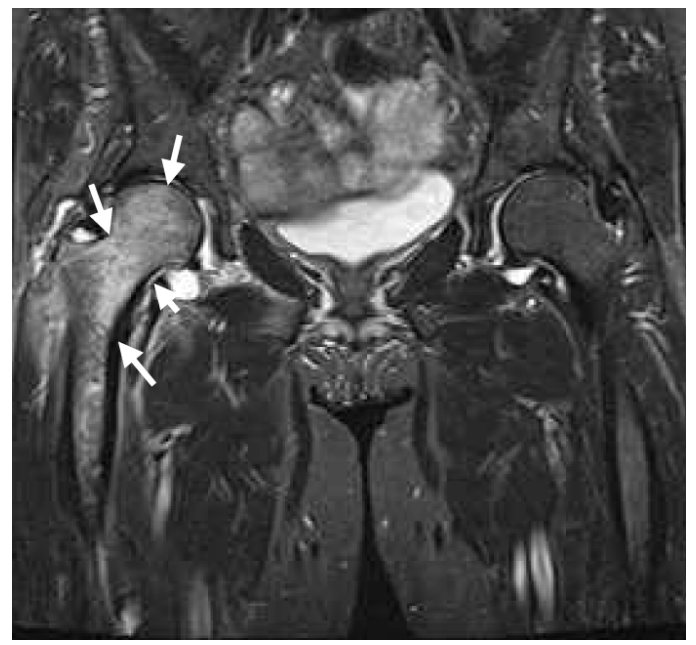

Fig. $(\mathbf{2 b}, \mathbf{c})$. Within a month after pain onset, hip MRI displays broad and heterogeneous low intensity in T1W (b) and high intensity in STIR (c) images. (d)

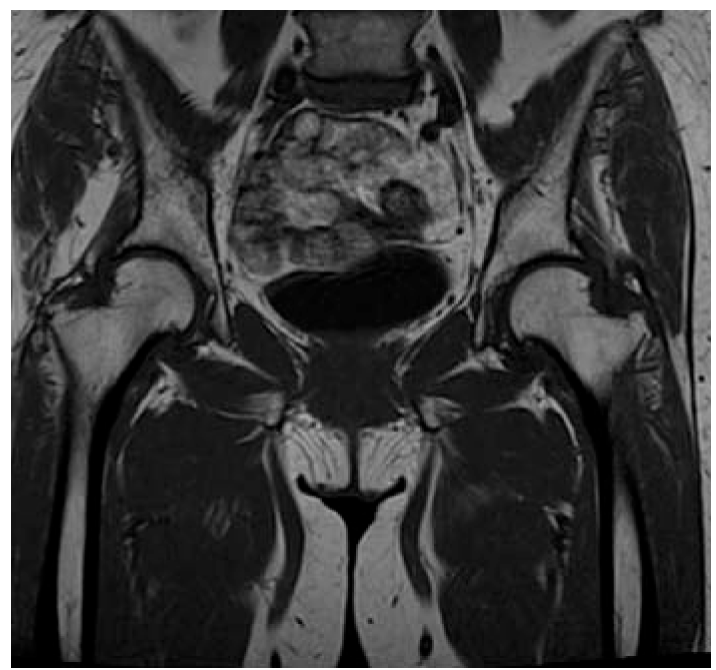

(e)

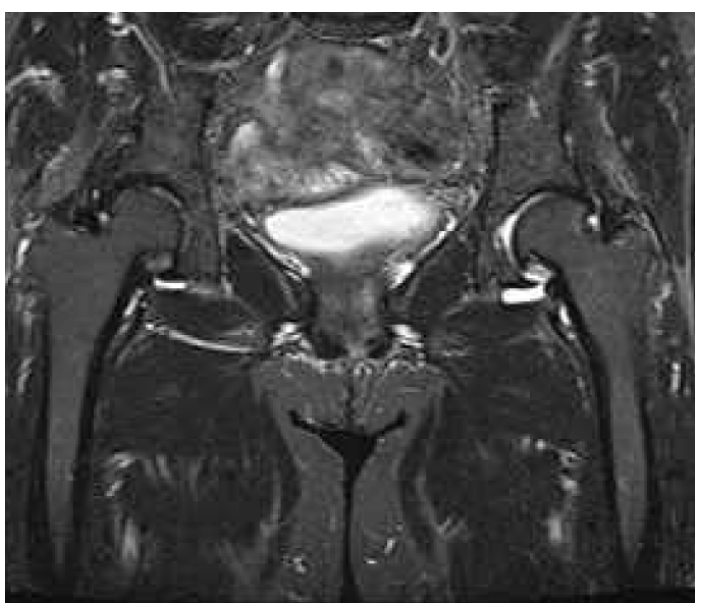

Fig. (2d, e). At more than 6 months after pain onset, MRI reveals no signal changes in the right femoral head in T1W (d) and STIR (e) images.

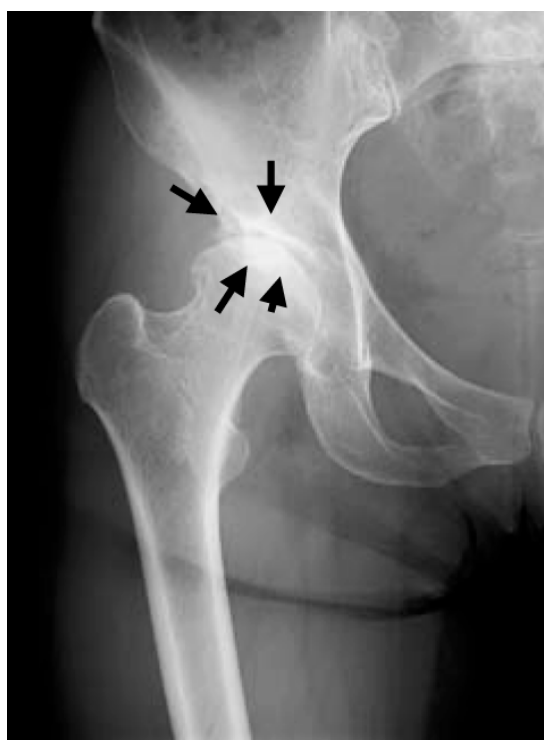

Fig. (2f). Plain radiograph taken as right hip joint pain persists showing KL grading II hip OA. Increased joint space narrowing is seen in the right hip joint (black arrows). 


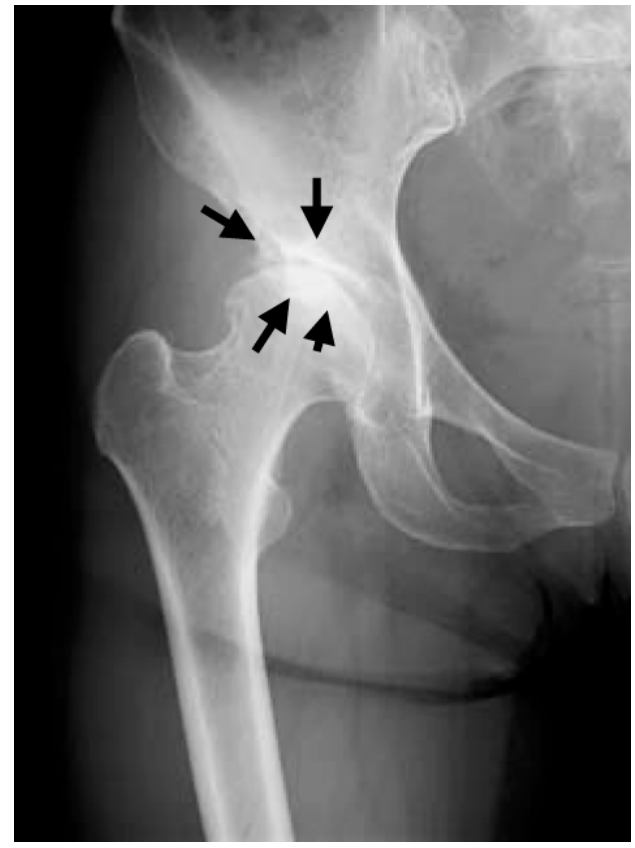

Fig. (3a). Plain radiograph taken at the onset of severe right hip joint pain showing KL grading III hip OA. Joint space narrowing, osteophytes, and osteosclerotic change are all evident in the right hip joint (black arrows).

(b)

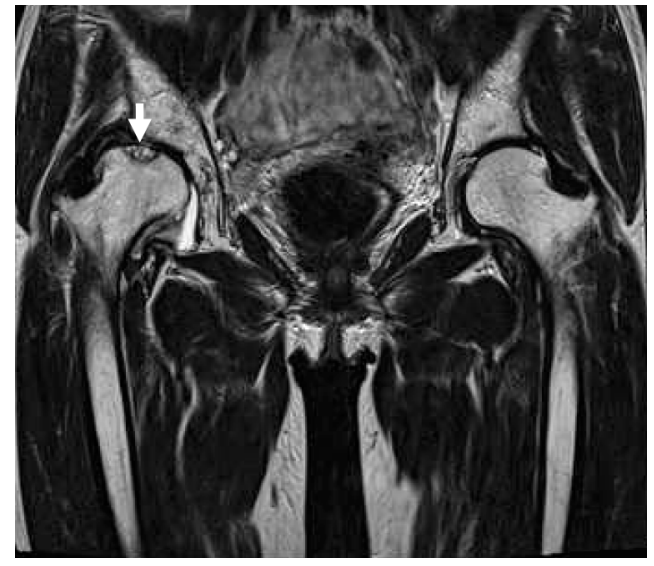

(c)

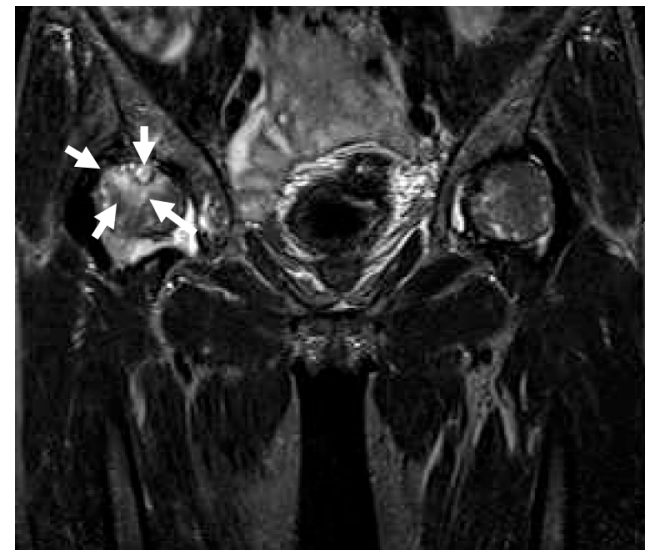

Fig. (3b, c). At more than 6 months after pain onset, MRI shows broad and heterogeneous low intensity in T1W (b) and high intensity in STIR (c) images (white arrows).

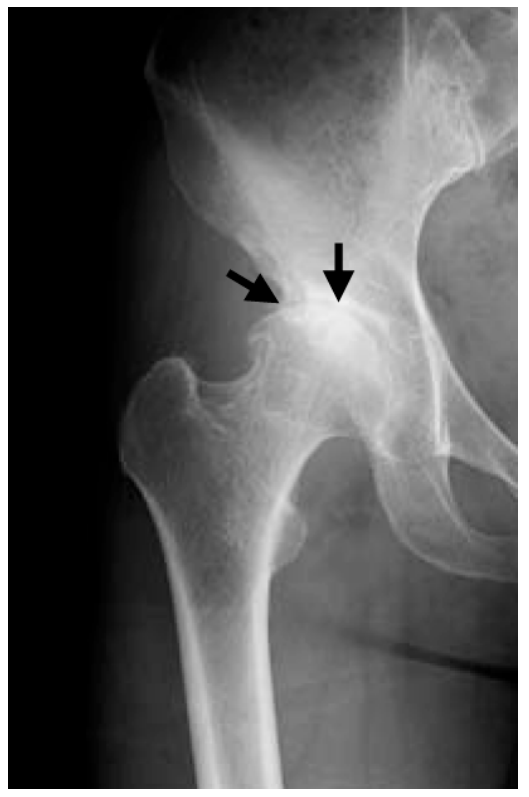

Fig. (3d). Plain radiograph taken after the right hip joint pain had disappeared showing KL grading IV hip OA. Joint space narrowing, osteophytes, osteosclerotic change, and hip joint deformity are all evident in the right hip joint (black arrows).

\section{DISCUSSION}

In this follow-up study, MRI bone signal changes were initially observed in the proximal femoral head of all hip OA patients with joint pain, regardless of KL grading. In Group A, MRI signal changes had disappeared in all but 1 patient (case 5) at the final follow-up a minimum of 6 months after pain onset. On the other hand, in Group B with persistent joint pain, all subjects continued to exhibit MRI bone changes at the study end point. OA progressed radiographically in all but 1 patient in both groups. Whereas OA worsened by $1 \mathrm{KL}$ grade at most in Group A, approximately $1 / 3$ of cases in Group B progressed by $2 \mathrm{KL}$ stages. Lastly, our study uncovered a significantly higher mean age for Group B.

Low intensity findings in $\mathrm{T} 1 \mathrm{~W}$ and high intensity findings in $\mathrm{T} 2 \mathrm{~W}$ images generally indicate fluid-related regions, including bone marrow edema, in bone [8]. In the present study, it was difficult to detect high intensity signals caused by bone marrow edema using $\mathrm{T} 2 \mathrm{~W}$ since bone marrow usually appears as a high intensity region due to adipose tissue. The STIR technique is therefore useful to provide better visualization of potentially relevant alterations, such as joint effusion, that are occasionally not visible in standard imaging sequences $[9,10]$. We routinely use STIR to detect fluid-related regions in bone marrow. It is our belief that abnormal bone signal changes should be evaluated using both T1W and STIR since the former displays weak low intensity signals in bone while the latter detects signal changes of fluid-related regions in bone marrow [8].

As described above, low intensity signals in $\mathrm{T} 1 \mathrm{~W}$ and high intensity signals in $\mathrm{T} 2 \mathrm{~W}$ images generally suggest bone marrow edema, but these findings are not specific to bone fracture. Karthiga et al. have reported that MRI is a helpful tool to detect bone marrow edema as a sign of recent fracture 
[11]. Taljanovic et al. compared MRI findings with histological results in patients with microfractures in different stages of healing after hip replacement surgery and concluded that the amount of bone marrow edema in the OA hip as evaluated by MRI correlated with the severity of pain, radiographic findings, and microfractures [12]. Guermazi et al. recently reviewed that MRI signal changes in joints frequently suggested microfractures [13]. In our previous study, advanced OA patients with joint pain exhibited MRI bone signal changes, whereas those without joint pain did not [4]. Here, joint pain initially occurred in accordance with bone alterations but had later disappeared in patients without bone affections at 6 months after onset. Thus, we consider MRI bone signal changes to be microfractures and the cause of joint pain.

A recent review described that cartilage was the main tissue affected by OA [14]. On the other hand, Burr and Gallant have proposed a hypothetical whole-joint model of OA progression in which they postulated that joint loading increases subchondral remodeling, probably to repair bone damage, and that bone alterations could primarily affect cartilage degeneration [15]. In this study, MRI bone alterations had reached the subchondral bone in all cases at pain onset. In 1 patient whose joint pain remained but bone signal pattern had changed, the affected lesions were localized in subchondral bone. Therefore, it is possible that the lesions detected by MRI were very near the articular cartilage and pathophysiologically affected it.

Group A included cases with advanced radiographic OA at pain onset (i.e., 2 patients with KL grading III). There was a tendency for MRI bone signal change and joint pain to disappear but radiographic OA to progress. On the other hand, no patients in Group $B$ exhibited advanced radiographic OA at onset. MRI bone signal changes and joint pain tended to persist in this group while radiographic OA worsened. According to the research on osteoarthritis against disability (ROAD) study of the elderly Japanese population of over 60 years of age, $47.0 \%$ of men and $70.2 \%$ of women had radiographic knee OA. However, the incidence of knee pain among knee OA patients was only $21.2 \%$ and $27.3 \%$ in men and women, respectively [7]. These results suggest that there are numerous OA patients who do not experience joint pain. According to a glucosamine/chondroitin arthritis intervention trial study, NSAIDs, such as celecoxib, relieved knee joint pain faster than glucosamine and/or chondroitin sulfate treatment. Very interestingly, joint pain had spontaneously improved at 24 weeks after onset in a nonmedicated placebo group [16]. These findings indicate that there are many patients with $\mathrm{OA}$ in whom joint pain naturally heals and cartilage degeneration does not necessarily cause pain, which supports our results that joint pain accompanying hip OA naturally heals in a large number of cases. In this study, the minimum observation period was relatively short at 6 months. However, we suspect that joint pain might have improved in more Group B cases if they had been observed longer.

The relationship between OA and osteoporosis is controversial. Im et al. have recently uncovered that OA is related to osteoporosis in cross-sectional and systematic analyses and that the risk of osteoporotic fracture does not decrease despite high $\mathrm{BMD}$ in patients with $\mathrm{OA}$, probably due to postural instability or inadequate muscle strength. Low BMD of the lumbar spine has been associated with a lower incidence of knee OA but does not arrest OA progression [17]. Bellido et al. described that the coexistence of osteoporosis and OA aggravated subchondral bone microstructural damage [18]. In this study, several patients had earlier been treated for osteoporosis, and therefore we could not precisely address the relationship between OA and BMD. In addition, the pathophysiology of osteoporotic fracture may be due not only to BMD, but also to bone quality [19], and so it remains challenging to associate the predisposition to fracture with low BMD even if the main pathophysiology of OA is microfracture.

As the ROAD study has shown, older age is an important risk factor for radiographic OA [20]. Here, the patients in Group B were significantly older than those in Group A. Moreover, no patients in Group A had progressed more than 1 radiographic OA stage by the study end point, in contrast with approximately $1 / 3$ of Group B patients whose condition worsened by $2 \mathrm{KL}$ stages. These findings imply that joints with bone alterations are predisposed to deteriorate more severely and rapidly in the elderly.

Recently, Yamamoto et al. proposed subchondral insufficiency fracture (SIF) as a new concept in femoral head collapse and stated that localized microfractures were often observed in the superficial subarticular bone in OA [21]. This evidence suggests that a portion of SIF and OA cases may be caused by microfracture. It has also been described that SIF develops into rapidly destructive coxopathy (RDC) radiographically [22]. Niimi et al. speculated that SIF was the cause of RDC and reported that the crescent sign in osteonecrosis $(\mathrm{ON})$ in plain radiographs was one of the features of RDC that resulted in subchondral microfracture [22]. Taljanovic et al. compared MRI features with histological findings in 19 hip OA subjects who had undergone total hip replacement surgery. They concluded that bone marrow edema detected by MRI in hip OA correlated with pain severity, radiographic findings, and microfrature [12]. Thus, their findings also support the notion that a percentage of OA cases are caused by microfracture. We very recently reported on a case in which a common pathophysiology among OA, SIF, ON, and RDC appeared to be responsible for microfracture [23]. Taken together, microfracture may contribute to the pathogenesis of numerous degenerative joint conditions.

Lastly, we earlier postulated the following pathophysiology, progression, and classification of OA: (1) MRI-based signal change stage: joint pain with or without early radiographic OA changes but with MRI signal changes, (2) remission stage OA: no or a slight degree of joint pain with radiographic OA but no or slight MRI bone alterations, and (3) end stage OA: joint pain with radiographic OA and significant MRI bone alterations [4]. In remission stage OA, joint pain typically improves and MRI findings vanish. Case 5 of Group A was considered to be an example of remission stage OA. However, hip MRI examination demonstrated a lesion around a cyst with osteosclerotic change, suggesting these bone signal alterations might have occurred in association with osteosclerosis. Therefore, it is possible that a pathophysiological condition accompanies osteosclerotic alterations in remission stage OA. On the other hand, some 
cases of radiographic end stage OA do not exhibit joint pain. Thus, it seems that while radiographic grading is not necessarily important, systematic image-based evaluation as well as the course of joint pain are required to diagnose remission stage $\mathrm{OA}$.

The limitations of this study are a relatively small cohort and short observation period. Further long-term studies of larger cohorts are needed to confirm our findings.

In conclusion, the present study indicates that 1) hip OA accompanied with joint pain has bone alterations, 2) these bone alterations disappear when joint pain improves, 3) bone alterations remain when joint pain continues, and 4) radiographic OA can progress to more advanced stage OA over a short time period. Therefore, the pathophysiology of $\mathrm{OA}$ and joint pain and the progression of OA may be due primarily to bone alterations.

\section{CONFLICT OF INTEREST}

The authors confirm that this article content has no conflict of interest.

\section{ACKNOWLEDGEMENTS}

We thank radiologists Dr. Hiroshi Koshihara and Dr. Tomoko Furukawa of Chushin Matsumoto Hospital for their invaluable assistance.

\section{REFERENCES}

[1] Moskowitz RW, Howell DS, Altman RD, Buckwalter JA, Goldberg VM. Osteoarthritis, $3^{\text {rd }}$ ed. Diagnosis and Medical/Surgical Management, Philadelphia: W.B. Saunders; 2003.

[2] Guermazi A, Niu J, Hayashi D, et al. Prevalence of abnormalities in knees detected by MRI in adults without knee osteoarthritis: population based observational study (Framingham Osteoarthritis Study). BMJ 2012; 345: e5339.

[3] Roemer FW, Guermazi A. MR imaging-based semiquantitative assessment in osteoarthritis. Radiol Clin North Am 2009; 47: 63354.

[4] Kamimura M, Nakamura Y, Ikegami S, UchiyamaS, Kato H. Joint pain undergoes a transition in accordance with signal changes of bones detected by MRI in hip osteoarthritis. Open Rheumatol J 2013; 7: 58-65.

[5] Kamimura M, Nakamura Y, Ikegami S, Mukaiyama K, Uchiyama $\mathrm{S}$, Kato $\mathrm{H}$. The pathophysiology of primary hip osteoarthritis may originate from bone alterations. Open Rheumatol J 2013; 7: 11218
[6] Denis F, Armstrong GW, Searls K, Matta L. Acute thoracolumbar burst fractures in the absence of neurologic deficit. A comparison between operative and nonoperative treatment. Clin Orthop Relat Res 1984; 189: 142-9.

[7] Kellgren JH, Lawrence JS. Radiologicalassessment of osteoarthrosis. Ann Rheum Dis 1957; 16: 494-502.

[8] Koff MF, Potter HG. Noncontrast MR techniques and imaging of cartilage. Radiol Clin North Am 2009; 47: 495-504.

[9] D'Aprile P, Tarantino A, Jinkins JR, Brindicci D. The value of fat saturation sequences and contrast medium administration in MRI of degenerative disease of the posterior/perispinal elements of the lumbosacral spine. Eur Radiol 2007; 17: 523-31.

[10] Genu A, Koch G, Colin D, Aho S, Pearson E, Ben Salem D. Factors influencing the occurrence of a T2-STIR hypersignal in the lumbosacral adipose tissue. Diagn Interv Imaging 2013. [Epub ahead of print].

[11] Karthiga S, Dubey S, Garber S, Watts R. Scurvy: MRI appearance. Rheumatology (Oxford) 2008; 47: 1109.

[12] Taljanovic MS, Graham AR, Benjamin JB, et al. Bone marrow edema pattern in advanced hip osteoarthritis: quantitative assessment with magnetic resonance imaging and correlation with clinical examination, radiographic findings, and histopathology. Skeletal Radiol 2008; 37: 423-31.

[13] Guermazi A, Roemer FW, Haugen IK, Crema MD, Hayashi D MRI-based semiquantitative scoring of joint pathology in osteoarthritis. Nat Rev Rheumatol 2013; 9; 236-51.

[14] Maldonado M, Nam J. The role of changes in extracellular matrix of cartilage in the presence of inflammation on the pathology of osteoarthritis. Biomed Res Int 2013; 2013: 284873.

[15] Burr DB, Gallant MA. Bone remodelling in osteoarthritis. Nat Rev Rheumatol 2012; 8: 665-73.

[16] Clegg DO, Reda DJ, Harris CL, et al. Glucosamine, chondroitin sulfate, and the two in combination for painful knee osteoarthritis N Engl J Med 2006; 354: 795-808.

[17] Im GI, Kim MK. The relationship between osteoarthritis and osteoporosis. J Bone Miner Metab 2013. [Epub ahead of print].

[18] Bellido M, Lugo L, Roman-Blas JA, et al. Subchondral bone microstructural damage by increased remodelling aggravates experimental osteoarthritis preceded by osteoporosis. Arthritis Res Ther 2010; 12(4): R152.

[19] Fonseca H, Moreira-Gonçalves D, Coriolano HJ, Duarte JA. Bone Quality: The determinants of bone strength and fragility. Sports Med 2013. [Epub ahead of print].

[20] Muraki S, Akune T, Oka H, et al. Incidence and risk factors for radiographic knee osteoarthritis and knee pain in Japanese men and women: a longitudinal population-based cohort study. Arthritis Rheum 2012; 64: 1447-56.

[21] Yamamoto T. Subchondral insufficiency fractures of the femoral head. Clin Orthop Surg 2012; 4: 173-80.

[22] Niimi R, Hasegawa M, Sudo A, Uchida A. Rapidly destructive coxopathy after subchondral insufficiency fracture of the femoral head. Arch Orthop Trauma Surg 2005; 125: 410-3

[23] Nakamura Y, Kamimura M, Mukaiyama K, Ikegami S, Uchiyama $\mathrm{S}$, Kato $\mathrm{H}$. Joint pain undergoes a transition in accordance with signal changes of bones detected by MRI in hip osteoarthritis. Distressed diagnosis of osteoarthritis, osteonecrosis, subchondral insufficiency fracture, or rapid destructive coxopathy -a case report. Open Rheumatol J 2014; 8: 20-3.

This is an open access article licensed under the terms of the Creative Commons Attribution Non-Commercial License (http://creativecommons.org/licenses/by-nc/ 3.0/) which permits unrestricted, non-commercial use, distribution and reproduction in any medium, provided the work is properly cited. 